\title{
Prevalence and factors associated with overweight or obesity among 2- to 6-year-old children in Hunan, China: a cross-sectional study
}

\author{
Na Liu ${ }^{1}$, Huixia Li ${ }^{2}$ Zhanjun Guo ${ }^{1}$, Xin Chen ${ }^{1}$, Peng Cheng ${ }^{1}$, Bian Wang ${ }^{1}$,

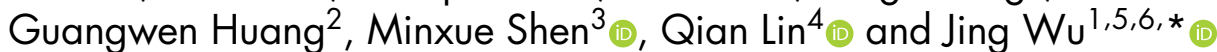 \\ 'Department of Endocrinology, Xiang-Ya Hospital, Central South University, Hunan Province 410008, People's \\ Republic of China: ${ }^{2}$ Department of Child Health Care, Hunan Provincial Maternal and Child Health Care Hospital, \\ Changsha, Hunan Province, People's Republic of China: ${ }^{3}$ Department of Social Medicine and Health Management, \\ Xiangya School of Public Health, Central South University, Changsha, Hunan Province, People's Republic of China: \\ ${ }^{4}$ Xiangya School of Public Health, Central South University, Hunan Province, People's Republic of China: ${ }^{5}$ Hunan \\ Engineering Research Center for Obesity and its Metabolic Complications, Changsha, Hunan, People's Republic of \\ China: ${ }^{6}$ National Clinical Research Center for Geriatric Disorders, Xiangya Hospital, Central South University, \\ Changsha, China
}

Submitted 30 December 2020: Final revision received 10 January 2022: Accepted 12 January 2022: First published online 17 January 2022

\begin{abstract}
Objective: To compare the prevalence of overweight or obesity (ow/ob) with WHO BMI cut-off points, International Obesity Task Force (IOTF) cut-off points and Chinese BMI criteria and examine its potential factors among preschool children in Hunan Province.

Design: A cross-sectional survey including anthropometric measurements and questionnaires about children's information, caregivers' socio-demographic characteristics and maternal characteristics. $\chi^{2}$ tests and univariate and multivariate binary logistic regression were performed to evaluate the possible factors of ow/ob.

Setting: Hunan, China, from September to October 2019.

Participants: In total, 7664 children 2 to 6 years of age.

Results: According to Chinese BMI criteria, about 1 in 7-8 children aged 2-6 years had ow/ob in Hunan, China. The overall estimated prevalence of ow/ob among 2- to 6-year-old children was significantly higher when based on the Chinese BMI criteria compared with the WHO BMI cut-off points and IOTF cut-off points. According to Chinese BMI criteria, ow/ob was associated with residing in urban areas, older age, male sex, eating snacking food more frequently, macrosomia delivery, caesarean birth, heavier maternal prepregnancy weight and pre-delivery weight.

Conclusion: The prevalence of ow/ob in preschool children in Hunan Province remains high. More ow/ob children could be screened out according to Chinese BMI cut-offs compared with WHO and IOTF BMI criteria. In the future, targeted intervention studies with matched controls will be needed to assess the long-term effects of intervention measures to provide more information for childhood obesity prevention and treatment.
\end{abstract}

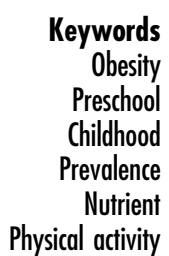

The prevalence of childhood overweight and obesity has risen in low- and middle-income countries during the past few decades ${ }^{(1,2)}$. The prevalence of obesity is related to genetic and biological factors, as well as socio-environmental factors, including family, school, community and national policy ${ }^{(3)}$. Childhood and adolescent obesity tracks adulthood obesity and has been implicated in many noncommunicable diseases, including type 2 diabetes, hypertension and $\mathrm{CVD}^{(1,4)}$. For most noncommunicable diseases caused by obesity, the risks depend partly on the age of onset and on the duration of obesity disease $^{(1)}$. Identifying modifiable risk and protective factors at earlier stages is critical to control obesity epidemics. 
BMI is a common indicator of body mass and nutritional status, which is widely used to classify overweight and obesity in adults. BMI as screening for ow/ob in children achieved the continuity in age with adult. The IOTF reference $^{(5)}$ and the WHO BMI criteria ${ }^{(6,7)}$ are the two most common international data sets used to define ow/ob in preschool children. In 2010, China established cut-off points of screening ow/ob for Chinese children and adolescents. The nutrition and health surveys of different diagnostic BMI criteria have reported large disparities in ow/ob rates in Chinese preschool children from different provinces: the overall prevalence of ow/ob ranges from $2.64 \%$ to $18.9 \%$, as reported in $2006-2017$ by IOTF criteria $^{(8-12)}$; the prevalence of obesity among 3- to 6-year ear-old children ranges from $15.9 \%$ to $19.9 \%$ as reported in 2014-2017 by the WHO BMI reference ${ }^{(13,14)}$ and the prevalence of ow/ob among children 3 to 6 years of age ranges from $22.47 \%$ and $26.0 \%$, as reported in 2017 by Chinese BMI criteria ${ }^{(10)}$. Owing to the heterogeneities in diagnostic criteria and the small sample size ${ }^{(10,14)}$, selfreported height and weight ${ }^{(10,14)}$ and unclear sampling method $^{(12,14)}$, these findings may not reflect the real prevalence of ow/ob.

Children's nutrition and lifestyles have changed significantly in China. WHO recognises that unhealthy dietary habits, low levels of physical activity (PA), socio-demographics, social and economic development are some of the possible factors for obesity ${ }^{(1)}$. There has been no large-scale survey of the prevalence and contributing factors of obesity in preschool children in Hunan, China, over the past decades. We collected children's general information, PA and dietary habits, caregivers' characteristics and socio-demographic characteristics. Dietary patterns consider overall diet rather than individual foods ${ }^{(15)}$; therefore, dietary patterns were derived from a FFQ. Therefore, the aim of the current study was to analyse the occurrence and potential factors of obesity with different criteria in Hunan Province in preschool-age children.

\section{Methods}

\section{Study design and population}

The current study is a cross-sectional survey conducted from September to October 2019 in Hunan Province. Inclusion criteria: (1) child's age is between 24 and 83 months; and (2) the child should have lived in that location for at least 1 year.

Subjects were selected by multistage stratified cluster sampling. The sample size calculation is given by

$$
\begin{gathered}
n=\frac{u_{\alpha / 2}^{2} \times \pi \times(1-\pi)}{\delta^{2}} \\
n^{\prime}=n \times \operatorname{deff}=n \times\left[1+\left(n_{j}-1\right) \times \rho\right]
\end{gathered}
$$

where $n$ is the sample size of simple randomised sampling, $\alpha$ is the inspection level, $\alpha=0.05 ; u$ is the boundary value of the standard normal distribution corresponding to $\alpha, u_{\alpha / 2}$ $=1.96 ; \pi$ is estimated prevalence of overweight and obesity, $\pi=0.05$ and $\delta$ is the allowable error, $\delta=0.008$. deff is the design effect, $n_{j}$ is the average group size, $n_{j}=56$ and $\rho$ is the correlation coefficient within the group, $\rho=0.03, n^{\prime}=7555 \cdot 65$. We estimated a rejection rate of $6 \%$, and the sample size needed 8009 children, considering that some children may refuse to participate or may not complete the questionnaires. The number of clusters is the total number of samples divided by the group size, that is, $8009 / 56=143 \cdot 02$. Finally, we sent out 8200 questionnaires from 144 clusters, detailed as follows. Hunan Province is located in the central south of China and is the home of fourteen cities. First, these cities were stratified into low, medium and high economic levels. Then, two cities from each economic level, two urban and two rural districts from each selected city, two streets and two towns from each selected district and three neighbourhood committees and three village committees from each selected town were randomly selected. In total, seventy-two neighbourhood communities and seventy-two village committees were selected. A consecutive sampling survey was used when selecting children from each neighbourhood committee/ village committee: the staff of the committee informed the children and caregivers of the local maternal and child health care centre for investigation at a predetermined time in each neighbourhood or village committee. The investigation was completed after the fifty-six children were investigated in order of arrival.

\section{Questionnaire and definition of variables}

We collected each child's information, including general information, such as gender, birth date, birth weight, mode of delivery, feeding practice for the first six months of life, screen time, time spent outdoors and dietary habits. Birth weights $<2500$ g, 2500-3999 g and $\geq 4000$ g were considered low birth weight, normal birth weight and macrosomia, respectively ${ }^{(16)}$. The feeding practices for the first six months were divided into exclusive breast-feeding, predominant breast-feeding, exclusive formula feeding and mixed feeding ${ }^{(17)}$ : exclusive breast-feeding meant the infant only fed on breast milk, but can be fed with oral rehydration salt, drops, syrups (vitamins, minerals, medicines); predominant breast-feeding meant the infant mainly fed on breast milk, but also took certain liquids (water and waterbased drinks, fruit juice), ritual fluids and oral rehydration salt, drops or syrups; mixed feeding meant the infant was fed with both breast milk and non-human milk and formula; formula feeding meant the infant was not fed with breast milk, but only with non-human milk and formula. Dietary habits were evaluated using a thirty-four-item FFQ for the past week based on the Dietary Guidelines for Chinese Residents and the eating habits of Hunan 
people. Food items were aggregated into eleven food groups based on similar nutrient content (online supplementary material, Supplemental Table 1) as follows: grains, cereals and other starchy staples; tofu and soy products; vegetables; fruits; algae; milk and dairy products; meat and poultry; aquatic; eggs; snacks and beverages, including infant formula (child-specific food items) and removing alcoholic beverages. In the self-reporting questionnaire, the mothers or caregivers were asked whether their children had consumed any of the food items and the frequency of consumption.

Socio-demographic characteristics included residence (urban or rural areas), economic level and parents or caregiver demographic characteristics (i.e. age in years, education and occupation). Maternal characteristics included pregnancy complications (hypertensive disorders of pregnancy, gestational diabetes mellitus, fetal distress and abnormal fetal position), prepregnancy weight, pre-delivery weight and gestational weight gain. Optimal weight gain during pregnancy was determined by the Institute of Medicine guidelines ${ }^{(18)}$ : underweight, normal weight, overweight and obese women should gain $12.5-18 \mathrm{~kg}$, $11.5-16 \mathrm{~kg}, 7-11.5 \mathrm{~kg}$ and $5-9 \mathrm{~kg}$ during pregnancy. The classification of prepregnancy BMI was based on the Chinese guidelines ${ }^{(19)}$ : underweight $\left(\mathrm{BMI}<18.5 \mathrm{~kg} / \mathrm{m}^{2}\right)$, normal weight (BMI $18.5-23.9 \mathrm{~kg} / \mathrm{m}^{2}$ ), overweight (BMI $24 \cdot 0-27.9 \mathrm{~kg} / \mathrm{m}^{2}$ ) and obese (BMI $\geq 28 \mathrm{~kg} / \mathrm{m}^{2}$ ).

\section{Anthropometric measurements}

All measurements were taken using the same type of apparatus and followed the same procedures. The children wore only light clothing, without shoes. Height was measured using a telescopic height measuring instrument to the nearest $0 \cdot 1 \mathrm{~cm}$. Weight was measured using a calibrated beam scale to the nearest $0.05 \mathrm{~kg}$. BMI was calculated as the weight divided by height squared $\left(\mathrm{kg} / \mathrm{m}^{2}\right)$.

\section{Overweight and obesity definition}

Under the WHO criteria, BMI for age $\mathrm{z}$ score (BAZ) between $2 \mathrm{SD}$ to $3 \mathrm{SD}$ and more than $3 \mathrm{SD}$ was defined as overweight and obesity for children aged 24-60 months ${ }^{(6)}$. BAZ between $1 \mathrm{SD}$ and $2 \mathrm{SD}$ and more than $2 \mathrm{SD}$ was defined as overweight and obesity for children aged 61-83 months ${ }^{(7)}$. The IOTF criteria for overweight and obesity are age and gender specific and based on centile curves that passing through BMI cut-off points for overweight $\left(25 \mathrm{~kg} / \mathrm{m}^{2}\right)$ and obesity $\left(30 \mathrm{~kg} / \mathrm{m}^{2}\right)$ at age $18^{(5)}$. The Chinese criteria define overweight and obesity based on centile curves that passing through BMI cut-off points for overweight $\left(24 \mathrm{~kg} / \mathrm{m}^{2}\right)$ and obesity $\left(28 \mathrm{~kg} / \mathrm{m}^{2}\right)$ at age 18 years $^{(20)}$.

\section{Data collection}

Each team consisted of nine data collectors and one supervisor, and everyone received two weeks of training. All study data were double-entered using Epidata3.1 (Jens M. Lauritsen, Michael Bruus, Odense, Denmark) and tested for concordance. For any inconsistent data, the original data were checked to ensure the high accuracy of the inputted data. The original questionnaire was kept in each survey area for verification by the research team.

\section{Statistical analyses}

Statistical analyses were performed using SPSS version 25.0 (IBM) and R, version 4.1.1 ${ }^{(21)}$. Frequencies and percentages were calculated for all categorical variables. Pearson's $\chi^{2}$ test was used to compare the prevalence of ow/ob based on different children's characteristics, socio-demographic characteristics and maternal characteristics. A univariable and multivariable logistic regression model was used to identify significant factors for ow/ob, starting with all variables with $P<0.05$ in the $\chi^{2}$ test. A $P$ value of $<0.05$ was considered to be statistically significant.

Dietary patterns were identified by factor analysis using the PCA, and varimax rotation was used to rotate the factors to better fit the data. Kaiser-Meyer-Olkin and Bartlett's test were used to evaluate the reliability and validity of the PCA. The Cronbach's alpha coefficient were used to evaluate the internal consistency of the dietary questionnaire. Dietary patterns were identified on the basis of the eigenvalue $(>2)$ and scree plot and named according to the foods with the highest loading on the pattern (factor loading of $>|0.30|$ ). We extracted two dietary patterns (see online supplementary material, Supplemental Table 2): the traditional meal pattern and snacking food pattern. The traditional meal pattern was loaded heavily on cereals, soy products, legume, vegetables, fruits, red meat, poultry, seafood, eggs and nuts, while the snacking food pattern was loaded heavily on processed meats, instant noodles, pastries, candy, nuts, fried and puffed food and beverages. Two components explained $14.3 \%$ and $5.7 \%$ of the variance, respectively. Factor scores were divided into four quartiles on the basis of their contribution to each pattern, and an increase from quartile (Q) 1 to Q4 was assumed. Exploratory factor analysis demonstrated the KaiserMeyer-Olkin test $(P=0.880)$ and Bartlett's test $\left(\chi^{2}=6845.089, P<0.001\right)$ supported the use of factor analysis. Cronbach's alpha coefficient (Cronbach's $\alpha=0.826$ ) indicates a good internal consistency of the dietary questionnaire.

The impact of missing data was also considered. Participants with missing data on outcomes - ow/ob (age, height, weight) - were excluded from the analyses. Twenty-two (0.3\%) children had a missing birth weight, $9(0.1 \%)$ were missing delivery mode, $2(<0.1 \%)$ were missing feeding practice for the first six months and 43 $(0.6 \%)$ were missing outdoor activity. Father's and mother's ages were missing for $155(2.0 \%)$ and 159 (2.1\%), respectively. A total of 145 (1.9\%) caregivers had a missing education and $170(2 \cdot 2 \%)$ were missing occupations. The 
missing values of hypertensive disorders of pregnancy, gestational diabetes mellitus, fetal distress, disposition, prepregnancy weight and pre-delivery weight were 178 (2.3\%), 170 (2.2\%), 187 (2.4\%), 169 (2.2\%), 18 (0.2\%) and $28(0.4 \%)$, respectively. On this basis, multiple imputation (mi) was performed using $\mathrm{R}$ with the chained equation package ${ }^{(22)}$. The analyses in Tables 1-3 (descriptive analysis, univariable and multivariable logistic regression) were performed with multiple imputation by the chained equations method. In addition, complete data were also used for univariable and multivariable logistic regression (see online supplementary material, Supplemental Table 3).

\section{Results}

\section{Prevalence of overweight and obesity among 2- to 6-year-old children}

In this survey, we distributed a total of 8200 questionnaires. A total of 463 children's data could not be evaluated because of refusal to participate or not completing the questionnaire, and thus we received 7737 (94.4\%) questionnaires. After excluding ineligible child age $(\geq 7$ years or $<2$ years) ( $n$ 17) and incomplete anthropometric measurements ( $n$ 56), the present analysis involved a total of 7664 children.

The estimated prevalence of overweight and obesity was significantly higher when based on the Chinese criteria (overweight: 9.1\%, 95\% CI: 8.8\%, 9.4\%; obesity: $4.3 \%$, $95 \%$ CI: $4.1 \%, 4.5 \%$ ) compared with the WHO (overweight: $4.5 \%$, $95 \%$ CI: $4.3 \%$, $4.7 \%$; obesity: $2 \cdot 1 \%$, $95 \%$ CI: $1.9 \%, 2.3 \%$ ) and IOTF (overweight: $4.6 \%, 95 \% \mathrm{CI}$ : $4.4 \%, 4 \cdot 8 \%$; obesity: $2 \cdot 0 \%, 95 \%$ CI: $1 \cdot 8 \%, 2 \cdot 1 \%$ ) cut-off points (Fig. 1). The number of ow/ob children screened using the WHO ( $n$ 507) and IOTF ( $n$ 506) criteria is about half that of those screened by Chinese criteria ( $n$ 1023).

\section{Population characteristics and univariate analysis of factors associated with ow/ob}

Tables 1 and 2 show the study population characteristics and univariate analysis of factors associated with ow/ob. The 72- to 83-month-old group had the fewest children, mainly because children over the age of 6 have basically entered primary school. Half of the children were reported to have been exclusive breastfed. Nearly half ( $42.9 \%)$ of the children were delivered by caesarean delivery (Table 1). Among the three categories of diagnostic standards, the estimated prevalence of ow/ob children under 60 months old was the lowest based on WHO criteria, and the estimated prevalence of ow/ob children aged 60-83 months was the lowest based on IOTF criteria. Ow/ob children were significantly older, had heavier birth weights, were more likely to live in urban areas and were delivered by caesarean delivery (Tables 1 and 2).

\section{Univariate and multivariate binary logistic analysis for potential ow/ob factors among children aged 2-6 years in Hunan, China}

Model covariates were age group (24-35 months, 36-47 months, 48-59 months, 60-71 months, 72-83 months); sex (girls, boys); children's birth weight (2500-3999 g, $<2500 \mathrm{~g}, \geq 4000 \mathrm{~g}$ ); mode of delivery (vaginal, caesarean); children's screen time ( $<1 \mathrm{~h} / \mathrm{d}, \geq 1 \mathrm{~h} / \mathrm{d})$, time spent outdoors ( $<1 \mathrm{~h} / \mathrm{d}, \geq 1 \mathrm{~h} / \mathrm{d}$ ), children's snacking food pattern (Q1, Q2, Q3, Q4); residence (rural, urban); father's age ( $<30$ years, $30-35$ years, $\geq 35$ years); mother's age $(<30$ years, 30-35 years, $\geq 35$ years); gestational diabetes mellitus (no, yes); prepregnancy weight ( $<50 \mathrm{~kg}, 50-55 \mathrm{~kg}, \geq 55$ $\mathrm{kg})$ and pre-delivery weight $(<65 \mathrm{~kg}, 65-70 \mathrm{~kg}, \geq 70 \mathrm{~kg})$.

Under logistic regression, crude OR and adjusted OR showed little difference between the two approaches (multiple imputation analysis (Table 3) and complete-case analysis (see online supplementary material, Supplemental Table 3)). Univariate and multivariate binary logistic regression analyses (Table 3) demonstrated the associations between significant factors and ow/ob using different references. Our results suggest that, according to Chinese BMI criteria, residence, sex, age, child birth weight, mode of delivery, time spent outdoors, maternal prepregnancy weight and pre-delivery weight and snacking food pattern were statistically significant variables. Children in the highest quartile (Q4) of the snacking food pattern had greater odds of ow/ob than those in the lowest quartile (Q1).

\section{Discussion}

BMI is the most commonly used simple measure of weight status, while there is not any consistent standard about childhood obesity. Two international criteria are used to define ow/ob for preschool children based on BMI: The IOTF reference is based on children under 25 years old from six national cross-sectional studies to define childhood overweight and obesity ${ }^{(5)}$ while the WHO published a growth standard for children aged $0-5^{(6)}$ and 5-19 years $^{(7)}$, which is based on samples of children who represent optimal growth. However, weight status in children and adolescents is complicated by racial and regional differences ${ }^{(23)}$. In 2010, the Chinese BMI cut-offs for screening ow/ob were established based on the BMI growth reference values ${ }^{(20)}$, which was obtained from two large national surveys of 69760 children. It has been gradually used in domestic epidemiological studies in recently years $^{(24-26)}$. We then employed the above three criteria (WHO, IOTF and Chinese criteria) to screen overweight and obesity. Our results show that the detection rate of ow/ob in preschool children is the highest by the Chinese criteria. The number of ow/ob children screened by Chinese criteria is about twice that screened by WHO or IOTF criteria. Similar trends have been reported, as demonstrated by a study from Asia, including China, Lebanon and 
Table 1 Children's characteristics and univariate analysis of factors associated with ow/ob

\begin{tabular}{|c|c|c|c|c|c|c|c|c|c|c|c|}
\hline \multirow[b]{2}{*}{ Parameters } & \multicolumn{2}{|l|}{ Total } & \multicolumn{3}{|c|}{ WHO criteria } & \multicolumn{3}{|c|}{ IOTF criteria } & \multicolumn{3}{|c|}{ Chinese criteria } \\
\hline & $n$ & $\%$ & $n$ & $\%$ & $\begin{array}{c}P- \\
\text { value }\end{array}$ & $n$ & $\%$ & $\begin{array}{c}P- \\
\text { value }\end{array}$ & $n$ & $\%$ & $\begin{array}{c}P- \\
\text { value }\end{array}$ \\
\hline Age group (month) & & & & & $<0.01$ & & & $<0.01$ & & & $<0.01$ \\
\hline $24-35$ & 1625 & $21 \cdot 2$ & 36 & $2 \cdot 2$ & & 46 & $2 \cdot 8$ & & 131 & $8 \cdot 1$ & \\
\hline $36-47$ & 1830 & 23.9 & 62 & $3 \cdot 4^{*}$ & & 85 & $4 \cdot 6^{*}$ & & 220 & $12 \cdot 0^{*}$ & \\
\hline $48-59$ & 1999 & $26 \cdot 1$ & 65 & 3.3 & & 126 & $6 \cdot 3^{*}, \dagger \dagger$ & & 270 & $13 \cdot 5^{\star}$ & \\
\hline $60-71$ & 1934 & $25 \cdot 2$ & 289 & 14.9*,†十,㧊 & & 217 & $11 \cdot 2^{*}$,十†,执 & & 345 & $17 \cdot 8^{\star}$,十七,执 & \\
\hline $72-83$ & 276 & 3.6 & 55 & $19 \cdot 9^{\star}, \dagger †, \ddagger \ddagger, \S \S$ & & 32 & $11 \cdot 6^{*}, \dagger †, \ddagger \ddagger$ & & 57 & $20 \cdot 7^{\star}, \dagger †$,拉 & \\
\hline Sex & & & & & $<0.01$ & & & 0.02 & & & $<0.01$ \\
\hline Boys & 3920 & $51 \cdot 1$ & 335 & 4.4 & & 285 & $7 \cdot 3$ & & 655 & $16 \cdot 7$ & \\
\hline Girls & 3744 & 48.9 & 172 & $2 \cdot 2$ & & 221 & 5.9 & & 368 & 9.8 & \\
\hline Children's birth weight (g) & & & & & $<0.01$ & & & $<0.01$ & & & $<0.01$ \\
\hline 2500-3999 & 6828 & $89 \cdot 1$ & 420 & $6 \cdot 2$ & & 424 & $6 \cdot 2$ & & 859 & $12 \cdot 6$ & \\
\hline$<2500$ & 277 & 3.6 & 14 & $5 \cdot 1$ & & 16 & $5 \cdot 8$ & & 25 & $9 \cdot 0$ & \\
\hline$\geq 4000$ & 559 & $7 \cdot 3$ & 73 & 13.1†,‡ & & 66 & $11.8 \dagger, \ddagger$ & & 139 & $24.9 \dagger, \ddagger$ & \\
\hline Mode of delivery & & & & & $<0.01$ & & & $<0.01$ & & & $<0.01$ \\
\hline Vaginal & 4372 & 57.0 & 248 & $5 \cdot 7$ & & 240 & $5 \cdot 5$ & & 522 & 11.9 & \\
\hline Caesarean & 3292 & $43 \cdot 0$ & 259 & 7.9 & & 266 & $8 \cdot 1$ & & 501 & $15 \cdot 2$ & \\
\hline $\begin{array}{l}\text { Feeding practice for the first six } \\
\text { months }\end{array}$ & & & & & $0 \cdot 15$ & & & 0.06 & & & 0.42 \\
\hline Exclusive breast-feeding & 3835 & $50 \cdot 0$ & 232 & $6 \cdot 0$ & & 224 & $5 \cdot 8$ & & 493 & $12 \cdot 9$ & \\
\hline Predominant breast-feeding & 1099 & $14 \cdot 3$ & 78 & $7 \cdot 1$ & & 80 & $7 \cdot 3$ & & 157 & $14 \cdot 3$ & \\
\hline Mixed feeding & 1953 & $25 \cdot 5$ & 134 & $6 \cdot 9$ & & 142 & $7 \cdot 3$ & & 259 & $13 \cdot 3$ & \\
\hline Exclusive formula feeding & 777 & $10 \cdot 1$ & 63 & $8 \cdot 1$ & & 60 & $7 \cdot 7$ & & 114 & $14 \cdot 7$ & \\
\hline Children's screen time $(\mathrm{h} / \mathrm{d})$ & & & & & $<0.01$ & & & $<0.01$ & & & $<0.01$ \\
\hline$<1$ & 3095 & $40 \cdot 4$ & 152 & 4.9 & & 162 & $5 \cdot 2$ & & 356 & 11.5 & \\
\hline$\geq 1$ & 4569 & $59 \cdot 6$ & 355 & $7 \cdot 8$ & & 344 & 7.5 & & 667 & 14.6 & \\
\hline Children's outdoor activity time (h/d) & & & & & 0.09 & & & 0.08 & & & $<0.01$ \\
\hline$<1$ & 3770 & $49 \cdot 2$ & 231 & $6 \cdot 1$ & & 230 & $6 \cdot 1$ & & 447 & 11.9 & \\
\hline$\geq 1$ & 3894 & $50 \cdot 8$ & 276 & $7 \cdot 1$ & & 276 & $7 \cdot 1$ & & 576 & $14 \cdot 8$ & \\
\hline Children's traditional meal pattern & & & & & 0.69 & & & 0.58 & & & 0.65 \\
\hline Q1 & 1916 & $25 \cdot 0$ & 129 & $6 \cdot 7$ & & 132 & $6 \cdot 9$ & & 247 & $12 \cdot 9$ & \\
\hline Q2 & 1916 & $25 \cdot 0$ & 116 & $6 \cdot 1$ & & 118 & $6 \cdot 2$ & & 268 & $14 \cdot 0$ & \\
\hline Q3 & 1916 & $25 \cdot 0$ & 134 & $7 \cdot 0$ & & 136 & $7 \cdot 1$ & & 262 & $25 \cdot 6$ & \\
\hline Q4 & 1916 & $25 \cdot 0$ & 128 & $6 \cdot 7$ & & 120 & $6 \cdot 3$ & & 246 & $12 \cdot 8$ & \\
\hline Children's snacking food pattern & & & & & $<0.01$ & & & 0.01 & & & $<0.01$ \\
\hline Q1 & 1916 & $25 \cdot 0$ & 112 & $5 \cdot 8$ & & 114 & 5.9 & & 216 & $11 \cdot 3$ & \\
\hline Q2 & 1916 & $25 \cdot 0$ & 111 & $5 \cdot 8$ & & 112 & $5 \cdot 8$ & & 250 & $13 \cdot 0$ & \\
\hline Q3 & 1916 & $25 \cdot 0$ & 117 & $6 \cdot 1$ & & 120 & $6 \cdot 3$ & & 258 & $13 \cdot 5 \|$ & \\
\hline Q4 & 1916 & $25 \cdot 0$ & 167 & ৪.7॥, १,§ & & 160 & ৪.4\|, १,, & & 299 & $15 \cdot 6 \|, \emptyset$ & \\
\hline
\end{tabular}

Ow/ob, overweight or obesity; Q, quartile. Data are $n(\%)$, and $P$-values are $\chi^{2}$ test.

${ }^{\star} P<0.05$ v. 24-35 months.

$\dagger P<0.05$ v. normal birth weight.

$\ddagger P<0.05 \mathrm{v}$. birth weight between 2500 and $3999 \mathrm{~g}$.

$\S P<0.05$ v. Q3

$\| P<0.05$ v. Q1.

$\eta P<0.05$ v. Q2.

†† $P<0.05$ v. 36-47 months.

抽<0.05 v. 48-59 months.

$\S \S P<0.05$ v. $60-71$ months.

Malaysia, showing that approximately one-third and half of obese Asian children aged 8-10 years were not screened using WHO and IOTF standards, respectively ${ }^{(27)}$. This suggests that the application of the Chinese criteria may be more appropriate to screen for obesity-related risks in Chinese children.

In our study, the prevalence of ow/ob among preschool children in Hunan was lower than those in other provinces of China. Compared with previous studies using WHO BMI criteria, the prevalence of ow/ob we surveyed $(7.8 \%$, age 3-6 years; $3 \cdot 3 \%$, age $3-4$ years; $15 \cdot 6 \%$, age 5-6 years) was significantly lower than that in Tianjin in 2014 (7.4\%, age
3-4 years; 35.0\%, age 5-6 years) ${ }^{(13)}$ and Beijing in 2017 ( $9.2 \%$, age $3-4$ years; $22 \cdot 1 \%$, age $5-6$ years $)^{(14)}$. The prevalence of obesity we surveyed was significantly lower than that of the China Health and Nutrition Survey in 2011 $(12.6 \% \text {, age } 3-6 \text { years })^{(9)}$, Liaoning Province in $2008-$ $2009(6.08 \% \text {, age 2-7 years })^{(11)}$ and Beijing in 2017 $(8.46 \% \text {, age } 3-6 \text { years })^{(10)}$ according to the IOTF criteria. Surprisingly, the obesity prevalence of preschool children living in the urban areas we reported (2.4\%, aged 2-6 years) was slightly lower than the National Epidemiological Survey on Simple Obesity in Childhood in $2006(2.64 \% \text {, age } 3-7 \text { years })^{(8)}$ by the IOTF criteria, 
Table 2 Socio-demographic and maternal characteristics and univariate analysis of factors associated with ow/ob

\begin{tabular}{|c|c|c|c|c|c|c|c|c|c|c|c|}
\hline \multirow[b]{2}{*}{ Parameters } & \multirow{2}{*}{$\begin{array}{c}\text { Total } \\
n\end{array}$} & \multirow[b]{2}{*}{$\%$} & \multicolumn{3}{|c|}{ WHO criteria } & \multicolumn{3}{|c|}{ IOTF criteria } & \multicolumn{3}{|c|}{ Chinese criteria } \\
\hline & & & $n$ & $\%$ & $P$-value & $n$ & $\%$ & $P$-value & $n$ & $\%$ & $P$-value \\
\hline Residence & & & & & $<0.01$ & & & $<0.01$ & & & $<0.01$ \\
\hline Urban & 3530 & $46 \cdot 1$ & 271 & $7 \cdot 7$ & & 281 & 8.0 & & 541 & $15 \cdot 3$ & \\
\hline Rural & 4134 & $53 \cdot 9$ & 236 & $5 \cdot 7$ & & 225 & 5.4 & & 482 & $11 \cdot 7$ & \\
\hline Economic level & & & & & 0.61 & & & 0.28 & & & 0.27 \\
\hline High & 2557 & 33.4 & 163 & $6 \cdot 4$ & & 155 & $6 \cdot 1$ & & 319 & $12 \cdot 5$ & \\
\hline Moderate & 2525 & 32.9 & 163 & $6 \cdot 5$ & & 166 & $6 \cdot 6$ & & 352 & 13.9 & \\
\hline Low & 2582 & 33.7 & 181 & $7 \cdot 0$ & & 185 & $7 \cdot 2$ & & 352 & $13 \cdot 6$ & \\
\hline Father's age (years) & & & & & $<0.01$ & & & $<0.01$ & & & $<0.01$ \\
\hline$<30$ & 1849 & $24 \cdot 1$ & 88 & $4 \cdot 8$ & & 85 & 4.6 & & 205 & $11 \cdot 1$ & \\
\hline $30-35$ & 3188 & 41.6 & 214 & $6 \cdot 7 \dagger$ & & 223 & $7.0 \dagger$ & & 452 & $14.2 \dagger$ & \\
\hline$\geq 35$ & 2627 & $34 \cdot 3$ & 205 & $7 \cdot 8 \dagger$ & & 198 & $7 \cdot 5 \dagger$ & & 366 & $13 \cdot 9 \dagger$ & \\
\hline Mother's age (years) & & & & & 0.02 & & & 0.13 & & & 0.47 \\
\hline$<30$ & 1579 & $20 \cdot 6$ & 85 & 5.4 & & 93 & 5.9 & & 196 & $12 \cdot 4$ & \\
\hline $30-35$ & 3385 & $44 \cdot 2$ & 218 & $6 \cdot 4$ & & 215 & $6 \cdot 4$ & & 460 & $13 \cdot 6$ & \\
\hline$\geq 35$ & 2700 & $35 \cdot 2$ & 204 & $7 \cdot 6 \ddagger$ & & 198 & $7 \cdot 3$ & & 367 & $13 \cdot 6$ & \\
\hline Main caregiver & & & & & 0.15 & & & 0.51 & & & 0.12 \\
\hline Mother & 5092 & $66 \cdot 4$ & 330 & 6.5 & & 331 & $6 \cdot 5$ & & 687 & 13.5 & \\
\hline Father & 214 & $2 \cdot 8$ & 22 & $10 \cdot 3$ & & 17 & 7.9 & & 28 & $13 \cdot 1$ & \\
\hline Grandparents & 2314 & $30 \cdot 2$ & 151 & 6.5 & & 153 & $6 \cdot 6$ & & 297 & $12 \cdot 8$ & \\
\hline Others & 44 & 0.6 & 4 & $9 \cdot 1$ & & 5 & 11.4 & & 11 & $25 \cdot 0$ & \\
\hline Caregiver's education & & & & & 0.85 & & & 0.94 & & & 0.96 \\
\hline Primary school or below & 1201 & $15 \cdot 7$ & 76 & $6 \cdot 3$ & & 77 & $6 \cdot 4$ & & 156 & $13 \cdot 0$ & \\
\hline Middle school & 2324 & $30 \cdot 3$ & 148 & 6.4 & & 151 & $6 \cdot 5$ & & 309 & $13 \cdot 3$ & \\
\hline High school & 2057 & $26 \cdot 8$ & 143 & $7 \cdot 0$ & & 143 & $7 \cdot 0$ & & 281 & $13 \cdot 7$ & \\
\hline University or above & 2082 & $27 \cdot 2$ & 140 & $6 \cdot 7$ & & 135 & $6 \cdot 5$ & & 277 & $13 \cdot 3$ & \\
\hline Caregiver's occupation & & & & & 0.49 & & & 0.08 & & & 0.25 \\
\hline Unemployed & 2295 & 29.9 & 140 & $6 \cdot 1$ & & 142 & $6 \cdot 2$ & & 298 & $13 \cdot 0$ & \\
\hline Manager & 751 & $9 \cdot 8$ & 61 & $8 \cdot 1$ & & 67 & 8.9 & & 120 & $16 \cdot 0$ & \\
\hline Technical staff & 1246 & $16 \cdot 3$ & 83 & $6 \cdot 7$ & & 79 & $6 \cdot 3$ & & 171 & $13 \cdot 7$ & \\
\hline Service works & 798 & 10.4 & 50 & $6 \cdot 3$ & & 42 & $5 \cdot 3$ & & 96 & $12 \cdot 0$ & \\
\hline Agricultural worker & 343 & 4.5 & 26 & $7 \cdot 6$ & & 22 & $6 \cdot 4$ & & 41 & $12 \cdot 0$ & \\
\hline Others & 2231 & $29 \cdot 1$ & 147 & $6 \cdot 6$ & & 154 & $6 \cdot 9$ & & 297 & $13 \cdot 3$ & \\
\hline Pregnancy complications & & & & & & & & & & & \\
\hline HDP & & & & & 0.38 & & & 0.07 & & & 0.35 \\
\hline Yes & 169 & $2 \cdot 2$ & 14 & $8 \cdot 3$ & & 17 & $10 \cdot 1$ & & 27 & $16 \cdot 0$ & \\
\hline No & 7495 & $97 \cdot 8$ & 493 & $6 \cdot 6$ & & 489 & 6.5 & & 996 & $13 \cdot 3$ & \\
\hline GDM & & & & & 0.06 & & & $<0.01$ & & & 0.02 \\
\hline Yes & 240 & $3 \cdot 1$ & 23 & $9 \cdot 6$ & & 28 & $11 \cdot 7$ & & 44 & $18 \cdot 3$ & \\
\hline No & 7424 & $96 \cdot 9$ & 484 & 6.5 & & 478 & 6.4 & & 979 & $13 \cdot 2$ & \\
\hline Fetal distress & & & & & $0 \cdot 19$ & & & 0.75 & & & 0.08 \\
\hline Yes & 151 & $2 \cdot 0$ & 6 & 4.0 & & 9 & $6 \cdot 0$ & & 13 & $8 \cdot 6$ & \\
\hline No & 7513 & $98 \cdot 0$ & 501 & $6 \cdot 7$ & & 497 & $6 \cdot 6$ & & 1010 & 13.4 & \\
\hline Abnormal fetal position & & & & & 0.71 & & & 0.52 & & & 0.93 \\
\hline Yes & 236 & $3 \cdot 1$ & 17 & $7 \cdot 2$ & & 18 & $7 \cdot 6$ & & 31 & $13 \cdot 1$ & \\
\hline No & 7428 & $96 \cdot 9$ & 490 & $6 \cdot 6$ & & 488 & $6 \cdot 6$ & & 992 & $13 \cdot 3$ & \\
\hline Pre-pregnancy weight (kg) & & & & & $<0.01$ & & & $<0.01$ & & & $<0.01$ \\
\hline$<50$ & 2735 & $35 \cdot 7$ & 123 & 4.5 & & 120 & 4.4 & & 256 & $9 \cdot 4$ & \\
\hline $50-55$ & 2399 & $31 \cdot 3$ & 153 & $6 \cdot 4 \|$ & & 137 & $5 \cdot 7 \|$ & & 310 & $12 \cdot 9 \|$ & \\
\hline$\geq 55$ & 2530 & 33.0 & 231 & $9 \cdot 1 \|, \emptyset$ & & 249 & $9 \cdot 8 \|, \emptyset$ & & 457 & $18 \cdot 1 \|, \emptyset$ & \\
\hline Pre-delivery weight (kg) & & & & & $<0.01$ & & & $<0.01$ & & & $<0.01$ \\
\hline$<65$ & 3434 & 44.8 & 151 & 4.4 & & 141 & $4 \cdot 1$ & & 312 & $9 \cdot 1$ & \\
\hline $65-70$ & 1876 & 24.5 & 129 & $6 \cdot 9^{*}$ & & 129 & $6 \cdot 9^{*}$ & & 257 & $13 \cdot 7^{\star}$ & \\
\hline$\geq 70$ & 2354 & 30.7 & 227 & $9 \cdot 6^{\star}, * \star$ & & 236 & $10 \cdot 0^{*},{ }^{* *}$ & & 454 & $19 \cdot 3^{*}, * \star$ & \\
\hline Pre-pregnancy BMI $\left(\mathrm{kg} / \mathrm{m}^{2}\right) \S$ & & & & & $<0.01$ & & & $<0.01$ & & & $<0.01$ \\
\hline$<18.5$ & 737 & $20 \cdot 9$ & 41 & $5 \cdot 6$ & & 40 & 5.4 & & 80 & $10 \cdot 9$ & \\
\hline $18.5-23.9$ & 2491 & $70 \cdot 6$ & 191 & $7 \cdot 7$ & & 198 & 7.9 & & 395 & $15 \cdot 9+\dagger$ & \\
\hline$\geq 24$ & 302 & $8 \cdot 6$ & 39 & 12.9††,㧊 & & 43 & 14.2††,㧊 & & 66 & 21.9††,㧊 & \\
\hline GWG & & & & & $<0.01$ & & & $<0.01$ & & & $<0.01$ \\
\hline Appropriate & 1379 & $39 \cdot 1$ & 88 & $6 \cdot 4$ & & 94 & $6 \cdot 8$ & & 194 & $14 \cdot 1$ & \\
\hline
\end{tabular}


Table 2 Continued

\begin{tabular}{|c|c|c|c|c|c|c|c|c|c|c|c|}
\hline \multirow[b]{2}{*}{ Parameters } & \multirow{2}{*}{$\begin{array}{c}\text { Total } \\
n\end{array}$} & \multirow[b]{2}{*}{$\%$} & \multicolumn{3}{|c|}{ WHO criteria } & \multicolumn{3}{|c|}{ IOTF criteria } & \multicolumn{3}{|c|}{ Chinese criteria } \\
\hline & & & $n$ & $\%$ & $P$-value & $n$ & $\%$ & $P$-value & $n$ & $\%$ & $P$-value \\
\hline Inadequate & 1028 & $29 \cdot 1$ & 71 & 6.9 & & 69 & $6 \cdot 7$ & & 121 & $11 \cdot 8$ & \\
\hline Excessive & 1123 & 31.8 & 112 & $10 \cdot 0 \ddagger \ddagger, \S \S,\|I\| \mid$ & & 118 & $10 \cdot 5 \ddagger \ddagger, \S \S,\|\|||$ & & 226 & $20 \cdot 1 \neq \ddagger, \S \S,\|\| \|$ & \\
\hline
\end{tabular}

Ow/ob, overweight or obesity; HDP, hypertensive disorders of pregnancy; GDM, gestational diabetes mellitus; GWG, gestational weight gain. Data are $n(\%)$, and $P$-values are $\chi^{2}$ test.

${ }^{*} P<0.05$ v. pre-delivery weight $<65 \mathrm{~kg}$

$\dagger P<0.05 v$. father's age $<30$ years old.

$\ddagger P<0.05 v$. mother's age $<30$ years old.

$\S$ Mothers in urban areas.

$\| P<0.05$ v. pre-pregnancy weight $<50 \mathrm{~kg}$.

$\eta P<0.05 \mathrm{v}$. pre-pregnancy weight between 50 and $55 \mathrm{~kg}$

${ }^{\star \star} P<0.05 \mathrm{v}$. pre-delivery between 65 and $70 \mathrm{~kg}$.

$\dagger \dagger P<0.05$ v. pre-pregnancy $\mathrm{BMl}<18.5 \mathrm{~kg} / \mathrm{m}^{2}$.

$\ddagger \ddagger P<0.05 \mathrm{v}$. pre-pregnancy BMl between 18.5 and $23.9 \mathrm{~kg} / \mathrm{m}^{2}$.

$\S \S P<0.05 v$. appropriate GWG.

$\|I\| P<0.05 v$. inadequate GWG.

representing the prevalence of childhood obesity in a Chinese city region. The prevalence of ow/ob we surveyed (14.8\%, age 3-6 years) was significantly lower than that in Changsha in $2017(26.0 \% \text {, age } 3-6 \text { years })^{(28)}$ and Beijing in 2017 (23.0\%, age 3-6 years) ${ }^{(10)}$ by Chinese BMI criteria. Beijing, Tianjin, Liaoning and Hunan per capita GDP ranked in the top 1, 3, 13 and 16 in China, ${ }^{(29)}$ respectively, and Changsha's GDP per capita 2018 ranked among the top of Hunan Province ${ }^{(30)}$. A national epidemiological survey on obesity in children under seven years of age in nine cities of China in 2016 found that the prevalence of obesity in the central region ( $4.4 \%$ ) was lower than that in the north $(4.7 \%)$ but higher than that in the south $(3.6 \%)^{(31)}$. The cities mentioned above (Beijing, Tianjin, Liaoning) are located in northern China, and Hunan is located in central China. We infer that the prevalence of childhood obesity in Hunan Province remains at a relatively low level compared with other urban areas in China, probably partly related to the lower macrosomia proportion (466/3176 v. 557/ $7664)^{(8)}$, lower caesarean delivery proportion $(537 / 1123$ v. 1123/7664) ${ }^{(14)}$, relatively lower level of economic development and geographic differences.

The prevalence of childhood obesity was higher in girls than in boys among European children aged 2-7 years ${ }^{(32)}$. However, our study shows that boys have a higher prevalence of obesity, corroborating other findings in China ${ }^{(9-11,13)}$. Gender disparity in obesity could be explained by numerous factors ${ }^{(33,34)}$ : Chinese girls are more likely to keep a slender shape, and boys spend more time playing computer games. In addition, Chinese families always think of the larger, more muscular ideal male body shape, and they are less likely to encourage sons to lose weight due to traditional gender preferences for boys. Therefore, Chinese families should enhance their feeding knowledge and pay more attention to children's weight status, especially boys.

In several developed areas, there was a reversal in urban-rural disparity, with the prevalence of overweight and obesity in rural children surpassing than that of their urban peer ${ }^{(35)}$. In our work, urban children were more likely to be obese than rural children. One possible explanation is that the rapid urbanisation and industrial development in China has led to changes in human activity and diet structure $^{(36)}$ : individuals living in urban areas have less occupational and daily PA that relies on motorised and labour-saving transport or appliances, and they consume greater amounts of high-energy-density foods than their rural counterparts; this excess energy is unlikely to be compensated through leisure physical activities.

Kindergarten Work Regulations ${ }^{(37)}$ pointed out that time spent outdoors for children (including outdoor sports activities) shall not be $<2 \mathrm{~h}$ a day. Our survey shows that nearly half of children spend less than $1 \mathrm{~h}$ outdoors and children who spend more time outdoors have a higher prevalence of ow/ob. Most researchers believe that increased PA could prevent weight gain and alleviate the obesity epidemic $^{(38,39)}$. More time outdoors does not connote more exercise, although outdoor space could provide the environment for $\mathrm{PA}^{(40,41)}$. The relationship between time spent outdoors and obesity needs to be further clarified by evaluating the time, intensity and form of PA in the future.

Dietary patterns consider overall diet rather than individual foods; therefore, they may better define to the public how to interpret or translate into diets and provide guidance for nutrition intervention and education ${ }^{(15)}$. We evaluated the effects of eating frequency of traditional meal and snack food on body weight and concluded that higher frequency of eating snack food was associated with ow/ob. Our study shows that the snacking food pattern was heavily loaded by the consumption of cakes, pastries, candy, fried food and beverages, which are rich in fats and sugar and low in fibre. A previous study showed that energy-dense snack food (e.g. sweets, cakes and pastries) consumption was associated with higher energy intake, poor nutrients and obesity ${ }^{(42)}$. Beverage consumption cannot reduce the intake of other foods because of its poor satiety compared 


\section{Public Health Nutrition}

Table 3 Univariate and multivariate logistic regression for ow/ob in Chinese children aged 2-6 years

\begin{tabular}{|c|c|c|c|c|c|c|c|c|c|c|c|c|}
\hline \multirow[b]{2}{*}{ Parameters } & \multicolumn{4}{|c|}{ WHO criteria } & \multicolumn{4}{|c|}{ IOTF criteria } & \multicolumn{4}{|c|}{ Chinese criteria } \\
\hline & cOR & $95 \% \mathrm{Cl}$ & aOR & $95 \% \mathrm{Cl} \dagger$ & cOR & $95 \% \mathrm{Cl}$ & aOR & $95 \% \mathrm{Cl} \dagger$ & $\mathrm{cOR}$ & $95 \% \mathrm{Cl}$ & aOR & $95 \% \mathrm{Cl}+$ \\
\hline \multicolumn{13}{|c|}{ Age group (month) } \\
\hline $24-35$ & 1 & & 1 & & 1 & & 1 & & 1 & & 1 & \\
\hline $36-47$ & 1.55 & $1 \cdot 02,2 \cdot 35^{\star}$ & 1.58 & $1.03,2.41^{*}$ & 1.67 & $1 \cdot 16,2 \cdot 41^{\star \star}$ & 1.74 & $1 \cdot 20,2 \cdot 54^{\star \star}$ & 1.56 & $1.24,1.96^{\star \star \star}$ & 1.59 & $1 \cdot 25,2 \cdot 01^{\star * \star}$ \\
\hline $48-59$ & 1.48 & $0.98,2.24$ & 1.51 & $0.99,2.32$ & $2 \cdot 31$ & $1.64,3.26^{\star \star \star}$ & 2.42 & $1.70,3.47^{\star \star \star *}$ & 1.78 & $1.43,2.22^{\star \star \star}$ & 1.84 & $1 \cdot 46,2 \cdot 32^{\star \star \star}$ \\
\hline $60-71$ & 7.75 & $5.45,11 \cdot 04^{\star \star \star}$ & $8 \cdot 25$ & $5 \cdot 68,11 \cdot 97^{\star \star \star}$ & $4 \cdot 34$ & $3 \cdot 13,6 \cdot 01^{\star \star \star}$ & 4.68 & $3 \cdot 31,6 \cdot 60^{\star \star \star}$ & 2.48 & $2 \cdot 00,3.06^{\star \star \star}$ & 2.56 & $2 \cdot 03,3 \cdot 22^{\star \star \star}$ \\
\hline $72-83$ & 10.99 & $7 \cdot 05,17 \cdot 11^{\star \star \star}$ & 11.98 & $7 \cdot 50,19 \cdot 14^{\star \star \star}$ & 4.50 & $2 \cdot 81,7 \cdot 21^{\star * *}$ & $4 \cdot 81$ & $2 \cdot 94,7 \cdot 88^{\star \star *}$ & 2.97 & $2 \cdot 11,4 \cdot 18^{\star \star \star}$ & $3 \cdot 19$ & $2 \cdot 22,4 \cdot 59^{\star \star \star}$ \\
\hline \multicolumn{13}{|l|}{ Sex } \\
\hline Girls & 1 & & 1 & & 1 & & 1 & & 1 & & 1 & \\
\hline Boys & 1.94 & $1 \cdot 61,2 \cdot 35^{\star \star \star}$ & 1.79 & $1 \cdot 47,2 \cdot 19^{\star \star \star}$ & 1.25 & $1.04,1.50^{*}$ & $1 \cdot 15$ & $0.95,1.38$ & 1.84 & $1 \cdot 61,2 \cdot 11^{\star \star \star}$ & 1.75 & $1.52,2 \cdot 01^{* * *}$ \\
\hline \multicolumn{13}{|c|}{ Children's birth weight (g) } \\
\hline $2500-3999$ & 1 & & 1 & & 1 & & 1 & & 1 & & 1 & \\
\hline$<2500$ & 0.81 & $0.47,1.40$ & 0.89 & $0.51,1.59$ & 0.93 & $0.55,1.55$ & 0.99 & $0.59,1.68$ & 0.69 & $0.45,1.05$ & 0.74 & $0.48,1.13$ \\
\hline$\geq 4000$ & $2 \cdot 29$ & $1 \cdot 76,2 \cdot 98^{\star \star \star}$ & 1.70 & $1 \cdot 27,2 \cdot 26^{\star \star \star}$ & 2.02 & $1.54,2 \cdot 66^{\star \star *}$ & 1.52 & $1 \cdot 14,2 \cdot 03^{\star \star}$ & $2 \cdot 30$ & $1 \cdot 87,2 \cdot 82^{\star \star *}$ & 1.76 & $1 \cdot 42,2 \cdot 18^{\star \star \star}$ \\
\hline \multicolumn{13}{|c|}{ Mode of delivery } \\
\hline Vaginal & 1 & & 1 & & 1 & & 1 & & 1 & & 1 & \\
\hline Caesarean & 1.42 & $1 \cdot 19,1 \cdot 70^{\star \star \star}$ & 1.25 & $1.03,1.51^{*}$ & 1.51 & $1 \cdot 26,1 \cdot 81^{* \star *}$ & 1.31 & $1.09,1.59^{\star \star}$ & 1.32 & $1 \cdot 16,1 \cdot 51^{\star \star \star}$ & $1 \cdot 19$ & $1.03,1.36^{\star}$ \\
\hline \multicolumn{13}{|c|}{ Children's screen time (h/d) } \\
\hline$<1$ & 1 & & 1 & & 1 & & 1 & & 1 & & 1 & \\
\hline$\geq 1$ & 1.63 & $1.34,1.98^{\star \star \star}$ & 1.03 & $0.84,1.28$ & 1.47 & $1 \cdot 22,1 \cdot 79^{\star * *}$ & 1.04 & $0.84,1.27$ & 1.32 & $1 \cdot 15,1.51^{\star \star *}$ & 1.02 & $0.88,1.18$ \\
\hline \multicolumn{13}{|c|}{ Children's outdoor activity time (h/d) } \\
\hline$<1$ & 1 & & 1 & & 1 & & 1 & & 1 & & 1 & \\
\hline$\geq 1$ & $1 \cdot 17$ & $0.98,1.40$ & $1 \cdot 12$ & $0.93,1.36$ & $1 \cdot 17$ & $0.98,1.41$ & 1.15 & $0.95,1.39$ & 1.29 & $1.13,1.47^{* * *}$ & 1.25 & $1.09,1.43^{* *}$ \\
\hline \multicolumn{13}{|c|}{ Children's snacking food pattern } \\
\hline Q1 & 1 & & 1 & & 1 & & 1 & & 1 & & 1 & \\
\hline Q2 & 0.99 & $0.76,1.30$ & 0.92 & $0.69,1.22$ & 0.98 & $0.75,1.28$ & 0.90 & $0.69,1.19$ & $1 \cdot 18$ & $0.97,1.43$ & $1 \cdot 15$ & $0.94,1.40$ \\
\hline Q3 & 1.05 & $0.80,1.37$ & 0.92 & $0.69,1.22$ & 1.06 & $0.81,1.38$ & 0.93 & $0.71,1.22$ & 1.23 & $1.01,1.49^{\star}$ & 1.14 & $0.93,1.39$ \\
\hline Q4 & 1.54 & $1 \cdot 20,1.97^{\star \star \star}$ & 1.35 & $1.04,1 \cdot 76^{\star}$ & 1.44 & $1 \cdot 12,1 \cdot 85^{\star \star *}$ & 1.23 & $0.95,1.59$ & 1.46 & $1 \cdot 21,1 \cdot 76^{\star \star \star}$ & 1.32 & $1.09,1.61^{\star *}$ \\
\hline \multicolumn{13}{|l|}{ Residence } \\
\hline Rural & 1 & & 1 & & 1 & & 1 & & 1 & & 1 & \\
\hline Urban & 1.37 & $1 \cdot 15,1.65^{\star \star \star}$ & 1.36 & $1.09,1 \cdot 70^{\star *}$ & 1.50 & $1.25,1 \cdot 80^{\star * *}$ & 1.47 & $1.18,1 \cdot 82^{\star * *}$ & 1.37 & $1 \cdot 20,1.57^{\star \star \star}$ & 1.37 & $1 \cdot 17,1 \cdot 60^{\star \star \star}$ \\
\hline \multicolumn{13}{|c|}{ Father's age (years) } \\
\hline$<30$ & 1 & & 1 & & 1 & & 1 & & 1 & & 1 & \\
\hline $30-35$ & 1.44 & $1 \cdot 12,1 \cdot 86^{* *}$ & 1.04 & $0.77,1.41$ & 1.56 & $1 \cdot 21,2 \cdot 02^{* * *}$ & $1 \cdot 26$ & $0.94,1.69$ & 1.33 & $1.11,1.58^{\star \star *}$ & $1 \cdot 11$ & $0.91,1.37$ \\
\hline$\geq 35$ & 1.69 & $1 \cdot 31,2 \cdot 19^{\star \star \star}$ & 0.95 & $0.65,1.39$ & 1.69 & $1 \cdot 30,2 \cdot 20^{\star \star *}$ & 1.07 & $0.73,1.55$ & $1 \cdot 30$ & $1.08,1.56^{\star \star}$ & 0.91 & $0.69,1.19$ \\
\hline \multicolumn{13}{|c|}{ Mother's age (years) } \\
\hline$<30$ & 1 & & 1 & & 1 & & 1 & & 1 & & 1 & \\
\hline $30-35$ & 1.21 & $0.94,1.57$ & 0.97 & $0.72,1.31$ & 1.08 & $0.84,1.39$ & 0.88 & $0.66,1.18$ & $1 \cdot 11$ & $0.93,1.33$ & 1.00 & $0.81,1.23$ \\
\hline$\geq 35$ & 1.44 & $1 \cdot 10,1 \cdot 87$ & 1.03 & $0.71,1.49$ & 1.26 & $0.98,1.63$ & 0.94 & $0.66,1.35$ & $1 \cdot 11$ & $0.92,1.34$ & 0.98 & $0.75,1.27$ \\
\hline \multicolumn{13}{|l|}{ GDM } \\
\hline No & 1 & & 1 & & 1 & & 1 & & 1 & & 1 & \\
\hline
\end{tabular}




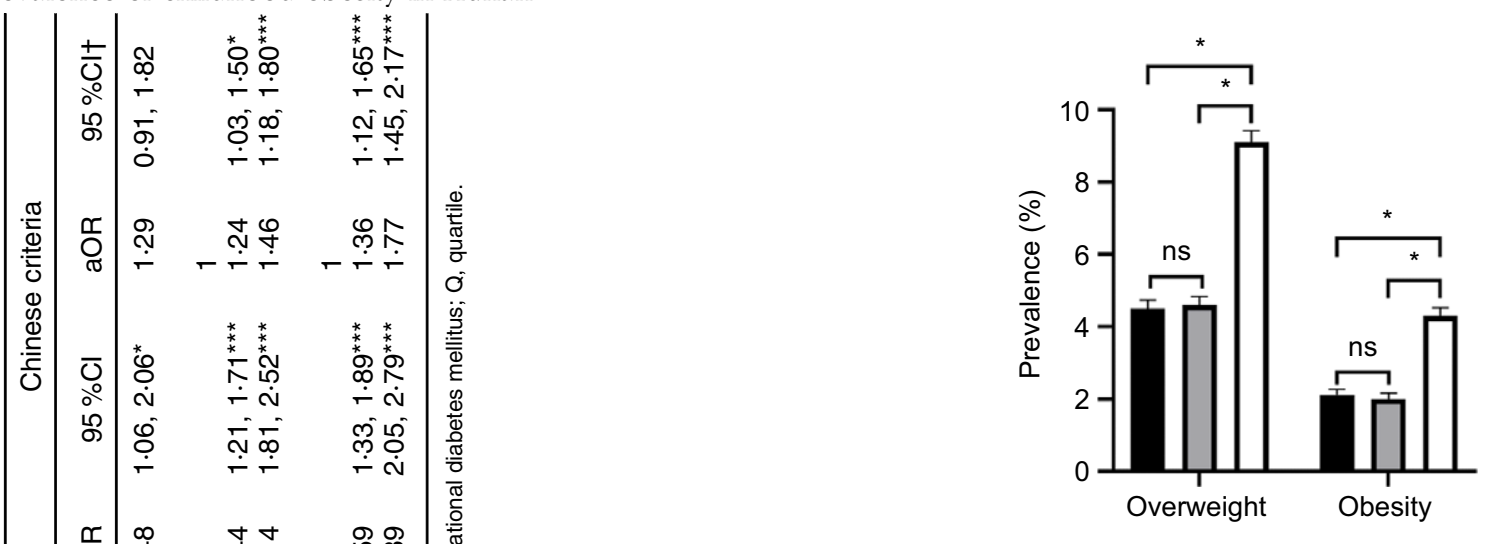

Fig. 1 Prevalence of overweight and obesity among 2- to 6year-old children in Hunan Province using the WHO, IOTF and Chinese BMI criteria. ${ }^{\star} P<0.05$. WHO criteria; $\square$, IOTF criteria; $\square$, Chinese criteria

with solid food ${ }^{(43)}$. The sugar content of most foods and beverages was considerably underestimated by parents ${ }^{(44)}$, providing easily accessible and practicable knowledge about sugar content may help parents choose healthier foods for their children. Caregivers and kindergarten teacher should encourage children to develop good eating habits and strictly limit snacks for children.

Previous research observed that the association between caesarean section and higher risk of offspring obesity attenuated but remained statistically significant after adjusting for maternal pre-pregnancy BMI ${ }^{(45)}$, which may be related to the different gastrointestinal microbiota established at birth for different delivery mode ${ }^{(46)}$. Maternal obesity may increase the risk of caesarean delivery and macrosomia ${ }^{(47)}$. Our study shows that children with caesarean birth and heavier maternal weights are more likely to suffer from obesity, although the heights of the mothers in rural areas were not collected. Next, the height of the mother can be collected to analyse the relationship between maternal obesity, caesarean section and obesity in offspring. Thus, we consider that it is wise that proper nutrition during pregnancy and prevention of excessive birth weight should not be overlooked in preventing childhood obesity.

Evidence shows that poverty and low socio-economic status may contribute to an epidemic of childhood and adolescent obesity ${ }^{(48)}$. In accordance with the previous findings reported, caregivers with lower education may contribute to the development of child ow/ob because they usually have less health knowledge, lower income, insecure food, etc. ${ }^{(49-51)}$. In the current study, we did not observe consistent associations between ow/ob and different economic levels, caregivers' education or occupation. Similarly, a study evaluated the impacts of different socio-economic indicators (such as parental education and occupation and socioeconomic status) on abdominal obesity and found that obesity is associated with socio-economic indicators in higher income countries, but this 
association was not observed in a lower-middle-income country, a possible reason for this discrepancy may be related to the different economic levels. Accordingly, a holistic approach that considers socio-economic background and family income and education will be helpful for better understanding the prevalence of overweight and obesity in children.

We know that the current study has several limitations. First, a cross-sectional study cannot verify causality. Second, the data collection on maternal weight was based on caregiver recall, which unavoidably led to recall bias, and the heights of the mother in rural areas were not collected, which could not be used to analyse the relationship between maternal BMI and offspring obesity. However, our analysis indicates that mothers who had heavier prepregnancy BMI or gestational weight gain were positively related to children's ow/ob in urban areas, which can partially explain the relationship between them. Finally, the use of a single frequency of food consumption for the past week was not adequate to account for the daily energy consumption, and the two dietary patterns explained only $20.0 \%$ of the total variance; thus, it is possible that other dietary patterns existed in the current study population. Nevertheless, this large-scale epidemiological survey involved 7664 children of age 2-6 years in Hunan Province. We employed three different criteria to define ow/ob simultaneously and identified several individual and socio-demographic factors, which is conducive to the early identification and intervention of overweight and obesity.

In conclusion, this large-scale epidemiological survey shows that the prevalence of ow/ob in preschool children in Hunan Province remains high. More ow/ob children could be screened out using Chinese BMI cut-offs compared with WHO and IOTF BMI criteria. Tailored interventions that encourage mothers to maintain healthy weight, choose vaginal delivery, increase health knowledge, create a healthy food environment to promote the development of healthy lifestyles for children, especially pay more attention to boys, may be important in reducing the risk of child ow/ob. In the future, targeted intervention studies with matched controls will be needed to assess the long-term effects of intervention measures to provide more information for childhood obesity prevention and treatment.

\section{Acknowledgements}

Acknowledgements: We thank the staff members at the Health Committee in Hunan for their guidance during the research project. We also thank the staff members of Maternity and Child Care Hospitals in Hunan selected children and their parents. Financial support: This work was supported by the project of the Hunan Health Committee (Grant No. 20201923), the Key Research \& Development Plan, Hunan, China (Grant No. 2020SK2066) and Science and Technology Program of Changsha, China (kh2003010). Conflict of interest: There are no conflicts of interest. Authorship: N.L. collected, analysed and interpreted the data and drafted the manuscript. Q.L., Z.G. and M.S. contributed to interpret the data and critically revised the manuscript for important intellectual content. X.C., C.P. and B.W. contributed to acquisition of data. H.L. and G.H. helped to design the study and recruited the participants. J.W. contributed to conception, design, interpretation of data and critically revised the manuscript for important intellectual content. All authors reviewed and approved the manuscript. Ethics of human subject participation: The current study was conducted according to the guidelines laid down in the Declaration of Helsinki, and all procedures involving research study participants were approved by the Ethics Committee of Xiang-Ya Hospital, Central South University on 23 September 2019, under the number 201909876. Written informed consent was obtained from all parents or guardians of the children.

\section{Supplementary material}

For supplementary material/s referred to in this article, please visit https://doi.org/10.1017/S136898002200012X

\section{References}

1. World Health Organization (2016) Childhood Overweight and Obesity. http://www.who.int/dietphysicalactivity/ childhood/en/ (accessed September 2020).

2. Pan XF, Wang L \& Pan A (2021) Epidemiology and determinants of obesity in China. Lancet Diabetes Endocrinol 9, 373392.

3. Larque E, Labayen I, Flodmark CE et al. (2019) From conception to infancy - early risk factors for childhood obesity. Nat Rev Endocrinol 15, 456-478.

4. GBD 2015 Obesity Collaborators, Afshin A, Forouzanfar MH et al. (2017) Health effects of overweight and obesity in 195 countries over 25 years. $N$ Engl J Med 377, 13-27.

5. Cole TJ, Bellizzi MC, Flegal KM et al. (2000) Establishing a standard definition for child overweight and obesity worldwide: international survey. BMJ 320, 1240-1243.

6. World Health Organization (2006) WHO Child Growth Standards: Methods and Development. https://www.who. int/childgrowth/standards/technical_report/en/ (accessed September 2020).

7. World Health Organization (2007) Development of a WHO Growth Reference for School-Aged Children and Adolescents. https://www.who.int/nutrition/publications/ growthref_who_bulletin/en/ (accessed September 2020).

8. Zong XN, Li H \& Zhang YQ (2015) Family-related risk factors of obesity among preschool children: results from a series of national epidemiological surveys in China. BMC Public Health 15, 927. 
9. Piernas C, Wang D, Du S et al. (2015) The double burden of under- and overnutrition and nutrient adequacy among Chinese preschool and school-aged children in 2009-2011. Eur J Clin Nutr 69, 1323-1329.

10. Liu S, Zhang J, Ma J et al. (2019) Synergistic interaction between bedtime and eating speed in predicting overweight and obesity in Chinese preschool-aged children. Aging 11, 2127-2137.

11. Ma YN, Chen T, Wang D et al. (2011) Prevalence of overweight and obesity among preschool children from six cities of northeast China. Arch Med Res 42, 633-640.

12. Zhou L, He G, Zhang J et al. (2011) Risk factors of obesity in preschool children in an urban area in China. Eur J Pediatr 170, 1401-1406.

13. Xiao Y, Qiao Y, Pan L et al. (2015) Trends in the prevalence of overweight and obesity among Chinese preschool children from 2006 to 2014. PLOS ONE 10, e0134466.

14. Liu S, Lei J, Ma J et al. (2020) Interaction between delivery mode and maternal age in predicting overweight and obesity in 1123 Chinese preschool children. Ann Transl Med 8, 474 .

15. Hu FB (2002) Dietary pattern analysis: a new direction in nutritional epidemiology. Curr Opin Lipidol 13, 3-9.

16. Gui YH \& Xue XD (2015) Pediatrics, 3rd ed. Beijing, China: People's Medical Publishing House.

17. WHO (2008) Indicators for Assessing Infant and Young Child Feeding Practices Part 1 Definitions. Conclusions of a Consensus Meeting Held 6-8 November 2007 in Washington DC, USA. https://www.who.int/publications/i/ item/9789241596664 (accessed July 2021).

18. Institute of $M \&$ National Research Council Committee to Reexamine IOMPWG (2009) The National Academies Collection: reports funded by National Institutes of Health. In Weight Gain During Pregnancy: Reexamining the Guidelines [KM Rasmussen \& AL Yaktine, editors]. Washington, DC: National Academies Press. https://www. ncbi.nlm.nih.gov/books/NBK32799/ (accessed January 2022).

19. Chen C \& Lu FC (2004) The guidelines for prevention and control of overweight and obesity in Chinese adults. Biomed Environ Sci: BES 17, 1-36.

20. Li H, Zong XN, Ji CY et al. (2010) Body mass index cut-offs for overweight and obesity in Chinese children and adolescents aged 2-18 years. Zhonghua liu xing bing xue za zhi= Zhonghua liuxingbingxue zazhi 31, 616-620.

21. R Project (2021) The R Project for Statistical Computing: A Language and Environment for Statistical Computing. https://www.r-project.org/ (accessed November 2021).

22. Zhang Z (2016) Multiple imputation with multivariate imputation by chained equation (MICE) package. Ann Transl Med 4, 30 .

23. Prentice AM \& Jebb SA (2001) Beyond body mass index. Obes Rev 2, 141-147.

24. Zhang L, Chen J, Zhang J et al. (2021) Regional disparities in obesity among a heterogeneous population of Chinese children and adolescents. JAMA Netw Open 4, e2131040.

25. Fu Y, Gou W, Hu W et al. (2020) Integration of an interpretable machine learning algorithm to identify early life risk factors of childhood obesity among preterm infants: a prospective birth cohort. BMC Med 18, 184.

26. Zhou X, Xue H, Duan R et al. (2015) The cross-sectional association of energy intake and dietary energy density with body composition of children in Southwest China. Nutrients 7, 5396-5412.

27. Liu A, Byrne NM, Kagawa M et al. (2011) Ethnic differences in the relationship between body mass index and percentage body fat among Asian children from different backgrounds. Br J Nutr 106, 1390-1397.
28. Ji M, Tang A, Zhang Y et al. (2018) The relationship between obesity, sleep and physical activity in Chinese preschool children. Int J Environ Res Public Health 15, 527.

29. National Bureau of Statistics of China (2019) The GDP per Capita in Each Province of China 2018. http://www.stats. gov.cn/tjsj/ndsj/2019/indexch.htm (accessed September 2020).

30. Hunan Provincial Bureau of Statistics Hunan Survey Team \& National Bureau of Statistics (2019) The Hunan Statistical Yearbook. http://222.240.193.190/19tjnj/indexch.htm (accessed September 2020).

31. Capital Institute of P \& Coordinating Study Group of Nine Cities on the Physical G Development of C (2018) A national epidemiological survey on obesity of children under seven years of age in nine cities of China in 2016. Zhonghua Er Ke Za Zhi 56, 745-752.

32. Garrido-Miguel M, Oliveira A, Cavero-Redondo I et al. (2019) Prevalence of overweight and obesity among European preschool children: a systematic review and meta-regression by food group consumption. Nutrients $\mathbf{1 1}$, 1698.

33. Song Y, Wang HJ, Ma J et al. (2013) Secular trends of obesity prevalence in urban Chinese children from 1985 to 2010: gender disparity. PLOS ONE 8, e53069.

34. Ricciardelli LA \& McCabe MP (2001) Children's body image concerns and eating disturbance: a review of the literature. Clin Psychol Rev 21, 325-344.

35. Sjöberg A, Moraeus L, Yngve A et al. (2011) Overweight and obesity in a representative sample of schoolchildren exploring the urban-rural gradient in Sweden. Obes Rev 12, 305-314.

36. Gong P, Liang S, Carlton EJ et al. (2012) Urbanisation and health in China. Lancet (London, England) 379, 843-852.

37. Ministry of Education of the People's Republic of China (2016) Kindergarten Working Rules. http://www.moe.gov. cn/srcsite/A02/s5911/moe_621/201602/t20160229_231184. html (accessed September 2020).

38. Wilkin T \& Voss LD (2004) Physical activity in young children. Lancet 363, 1162-1163.

39. Jakicic JM \& Otto AD (2006) Treatment and prevention of obesity: what is the role of exercise? Nutr Rev 64, S57-61.

40. Shanahan DF, Franco L, Lin BB et al. (2016) The benefits of natural environments for physical activity. Sports Med $\mathbf{4 6}$, 989-995.

41. Gray C, Gibbons R, Larouche R et al. (2015) What is the relationship between outdoor time and physical activity, sedentary behaviour, and physical fitness in children? A systematic review. Int J Environ Res Public Health 12, 6455-6474.

42. Andrieu E, Darmon N \& Drewnowski A (2006) Low-cost diets: more energy, fewer nutrients. Eur J Clin Nutr 60, 434-436.

43. Pan A \& Hu FB (2011) Effects of carbohydrates on satiety: differences between liquid and solid food. Curr Opin Clin Nutr Metab Care 14, 385-390.

44. Dallacker M, Hertwig R \& Mata J (2018) Parents' considerable underestimation of sugar and their child's risk of overweight. Int J Obes 42, 1097-1100.

45. Yuan C, Gaskins AJ, Blaine AI et al. (2016) Association between cesarean birth and risk of obesity in offspring in childhood, adolescence, and early adulthood. JAMA Pediatr 170, e162385.

46. Musso G, Gambino R \& Cassader M (2010) Obesity, diabetes, and gut microbiota: the hygiene hypothesis expanded? Diabetes Care 33, 2277-2284.

47. Ma RCW, Schmidt MI, Tam WH et al. (2016) Clinical management of pregnancy in the obese mother: before conception, during pregnancy, and post partum. Lancet Diabetes Endocrinol 4, 1037-1049. 
48. Demment MM, Haas JD \& Olson CM (2014) Changes in family income status and the development of overweight and obesity from 2 to 15 years: a longitudinal study. BMC Public Health 14, 417.

49. Tang A, Ji M, Zhang Y et al. (2018) Dietary behaviors and caregiver perceptions of overweight and obesity among Chinese preschool children. Int J Environ Res Public Health 15, 716.
50. Adler NE \& Stewart J (2010) Health disparities across the lifespan: meaning, methods, and mechanisms. Ann N Y Acad Sci 1186, 5-23.

51. Leon Guerrero RT, Barber LR, Aflague TF et al. (2020) Prevalence and predictors of overweight and obesity among young children in the children's healthy living study on Guam. Nutrients 12, 2527. 